\title{
Remote sensing methods for biomass estimation of green algae attached to nursery-nets and raft rope
}

\author{
Xiaopeng Jiang ${ }^{\mathrm{a}, \mathrm{c}}$, Zhiqiang Gao ${ }^{\mathrm{a}, *}$, Qingchun Zhang ${ }^{\mathrm{b}, \mathrm{d}, \mathrm{e}}$, Yueqi Wang ${ }^{\mathrm{a}}$, Xinpeng Tian ${ }^{\mathrm{a}}$, \\ Weitao Shang ${ }^{\mathrm{a}, \mathrm{c}}$, Fuxiang $\mathrm{Xu}^{\mathrm{f}}$ \\ ${ }^{a}$ Yantai Institute of Coastal Zone Research, Chinese Academy of Sciences, Yantai, 264003, China \\ ${ }^{\mathrm{b}}$ CAS Key Laboratory of Marine Ecology and Environmental Sciences, Institute of Oceanology, Chinese Academy of Sciences, Qingdao, 266071, China \\ ${ }^{\mathrm{c}}$ University of Chinese Academy of Sciences, Beijing, 100049, China \\ ${ }^{\mathrm{d}}$ Laboratory for Marine Ecology and Environmental Science, Qingdao National Laboratory for Marine Science and Technology, Qingdao, 266071, China \\ ${ }^{\text {e }}$ Center for Ocean Mega-Science, Chinese Academy of Sciences, Qingdao, 266071, China \\ ${ }^{\mathrm{f}}$ School of Public Administration, Shandong Technology and Business University, Yantai, 264005, China
}

\section{A R T I C L E I N F O}

\section{Keywords:}

Green tide

Biomass estimation

Unmanned aerial vehicle

Attached algae

Subei shoal

\begin{abstract}
A B S T R A C T
Accurate estimation of the biomass of raft-attached green algae is important for predicting the scale of greentides in the Yellow Sea, China. In this study, two different biomass estimation methods are proposed: green algae attached to nursery-net (GAAN) and green algae attached to rope (GAAR). The GAAN method involves the use of images obtained using an unmanned aerial vehicle (UAV), high-resolution satellite images, and data from a statistical yearbook. The GAAR method uses high-resolution satellite images and data from a field sample survey. The results showed that the biomass of GAAN and GAAR in the Subei Shoal during 2017 was 8868 tons and 2974 tons respectively. A longer-term study of the biomass of GAAN and GAAR could provide quantitative information for the earnings forecasts of Porphyra yezoensis and for green-tide prevention.
\end{abstract}

\section{Introduction}

Green tides, a form of harmful algae blooms, have outbroken continuously in the Yellow Sea, China for 12 years since 2007 (Zhou et al., 2015). The agglomeration and decomposition of large amounts of green-tide algal biomass have destroyed the marine ecosystem in the immediate area of blooms (Nelson et al., 2008). This has caused serious damage to the scenery, environment, and mariculture industry along the south coast of the Shandong peninsula, China, and has also resulted in great economic loss (Ye et al., 2011; Victor and Adriana, 2013; Yu et al., 2018). Many previous studies have illustrated that the initial biomass of the Yellow Sea green-tide came from green algae attached to aquaculture rafts used to culture Porphyra yezoensis in the Subei Shoal (Liu et al., 2009; Zhang et al., 2015; Huo et al., 2016), which was an important $P$. yezoensis aquaculture base in China (Liu et al., 2013b). $P$. yezoensis is an edible sea alga, which is rich in protein, vitamins and minerals, and other nutrients. It has been an important economic cultivation sea algae in Jiangsu province (Lu et al., 2018). Nevertheless, the growth of green algae has been one of the most difficult problems facing the $P$. yezoensis aquaculture industry. During the seedling stage, the filaments of $P$. yezoensis grow in oyster shells used for P.yezoensis breeding, and during this time some single-celled green algae can penetrate inside the shells to grow. During the growth stage, green algae can also attach to nursery-nets to compete with $P$. yezoensis, thus reducing the yield and quality of $P$. yezoensis (Shi and Wang, 1996). Therefore, the green algae attached to rafts have not only caused the Yellow Sea green-tide, which produced enormous losses and a negative social impact to the Shandong coastal cities, but have also significantly influenced the economic benefits of the $P$. yezoensis industry in Jiangsu province.

Green-tide biomass is an important parameter for the study of green-tide and disaster mitigation and prevention. Since the emergence of the Yellow Sea green-tide, research on its biomass estimation has been conducted. Accurate estimation of the floating green-tide biomass is of great significance to make the emergency response plan. Wang et al. (2015) studied the spatiotemporal distribution of floating green algae in the Yellow Sea during the spring of 2012. Xiao et al. (2017) constructed a surface feature spectral estimation model for biomass per unit area of floating green-tides on the sea surface based on field experiments and reported the related model estimations. Liu et al. (2015) adopted a biomass per unit area of floating green-tides obtained by horizontal trawling on the surface of Yellow Sea. The biomass was

\footnotetext{
* Corresponding author.

E-mail address: zqgao@yic.ac.cn (Z. Gao).
} 
multiplied by the area covered by green algae, which had been extracted from satellite data, as a means of estimating the total biomass and distribution features of the floating green-tides. In addition, since the raft-attached green algae provide initial biomass for the Yellow Sea green-tide, its biomass would be an important indicator that decides the magnitude of green-tide. Therefore, Zhang et al. (2018b) estimated that the total biomass into seawater could have amounted to $10000 \mathrm{t}$ (wet weight) in the Subei Shoal area in 2017. Xu et al. (2018) also proposed a biomass estimation model and estimated the biomass of green algae attached to rafts and raft ropes.

Current research on green algal biomass has focused on the period of massive floating on the sea surface. However, for the biomass estimations during the early stages of green-tides when the algae are still attached to rafts, the data used in such studies have mostly been subjective estimation data obtained by social surveys and other methods, and the results have only provided rough order of magnitude estimates with significant differences existing among studies. Algae attached to rafts have also largely been treated as an entity, without distinguishing between the green algae attached to nursery-nets (GAAN) and green algae attached to rope (GAAR). Additionally, there has been little research on the biomass of GAAN in the Subei Shoal.

The present study therefore aims to accurately monitor and estimate the biomass of GAAR and GAAN in the Subei Shoal green-tide algae source area of the Yellow Sea. The study used an unmanned aerial vehicle (UAV), satellite remote sensing, field sampling, a statistical yearbook to propose two biomass estimation methods: one for GAAR and one for GAAN. We anticipate that our findings will provide effective methods for the monitoring of green algal biomass attached to rafts, and will significantly enhance the capacity of earnings forecasts related to the $P$. yezoensis aquaculture industry, in addition to assisting towards the prevention and control of Yellow Sea green-tides.

\section{Materials and methods}

\subsection{Study area and field survey}

The field survey was undertaken in the Zhugensha sandbank $\left(121.2939^{\circ} \mathrm{E}, 32.7882^{\circ} \mathrm{N}\right)$ in the Subei Shoal (Fig. 1a and b), which experiences regular semidiurnal tides. The tidal flats are exposed above the water level during low tide periods, and are inundated during high tide periods. At the time of this study, semi-floating raft cultivation techniques were used for $P$. yezoensis aquaculture. Each raft unit was of the same specifications and size. Each raft unit consisted of one nurserynet, two pieces of rope, two transverse bamboo poles to fix the rope distance, and four erecting brackets to support the raft (Fig. 1c). The size of raft unit is shown in Fig. $6 \mathrm{~d}$.

Before the withdrawal of the rafts, biomass continued to increase with the growth of green algae. Therefore, the sampling time was close to the raft withdrawal time as a means of ensuring that the estimation of the initial biomass of green algae was more accurate. Sampling of green algae was conducted on the morning of May 11, 2017, which was same day as the raft withdrawal. Six columns of rafts were selected at random, from which, three ropes were chosen randomly from each column. A total of 18 ropes were therefore obtained, which were then sampled for green algae. Sampling was performed using a scraper to scrape off the green algae from $1 \mathrm{~m}$ lengths of each rope. Green algal samples were then placed into sample bags, labelled with numbers, and brought back to the research vessel. Samples were subsequently washed with seawater to remove sediment, and water was squeezed out after washing. Each of the 18 samples were weighed, and the mean wet weight $\left(\boldsymbol{W}_{\boldsymbol{g}}, \mathrm{g} / \mathrm{m}\right)$ of GAAR was calculated by summing the wet weight of all samples and dividing by the number of samples.

\subsection{UAV and satellite data collection and processing}

The DJI Inspire 1 (SZ DJI Technology Co., Ltd., Shenzhen, China) four-rotor UAV (Fig. 2a) was used in this study, and took off from and landed on the deck of the research vessel (Fig. 2b). The UAV was unaffected by clouds since it had a low flight altitude of $\sim 5 \mathrm{~m}$. The Inspire 1 UAV adopts both the global navigation satellite system (GLONASS) and the global positioning system (GPS), and the acquisition of aerial images used position information. Digital orthophoto maps were generated from the aerial images using photogrammetry software. The precision requirements for a small range of large-scale mapping has been applied and verified in previous studies (Wang et al., 2018; Zhang et al., 2018a).

Orthographic aerial photographing of two columns of aquaculture rafts in the survey area was conducted on March 30, 2017, by setting 61 selected rafts as targets. It was necessary to ensure that the forward overlap rate was $>70 \%$ and that the side overlap rate was $>40 \%$. Pix4Dmapper (Pix4D SA, Lausanne, Switzerland) was applied to generate the digital orthophoto images of the rafts (an example raft is shown in Fig. 4a). The UAV was also used to carry out an investigation of the growth status in the nearshore aquaculture zone, as well as the
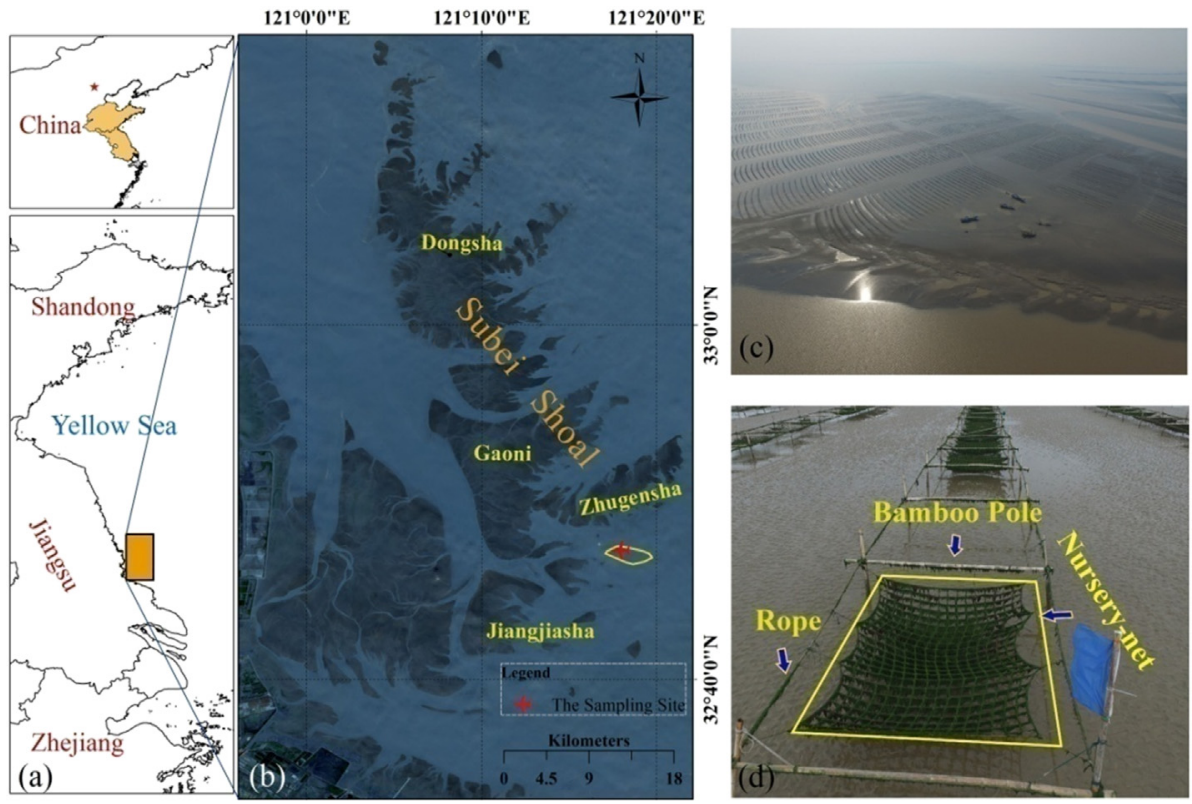

Fig. 1. Survey area and $P$. yezoensis aquaculture rafts in the Subei Shoal: (a) the Subei Shoal area in the Yellow Sea (indicated by a orange rectangle); (b) the Survey area (indicated by the yellow outline) and some sandbanks within the Subei Shoal; (c) unmanned aerial vehicle image of survey area; (d) an example of a $P$. yezoensis aquaculture raft and its components including ropes and a nursery-net. (For interpretation of the references to color in this figure legend, the reader is referred to the Web version of this article.) 

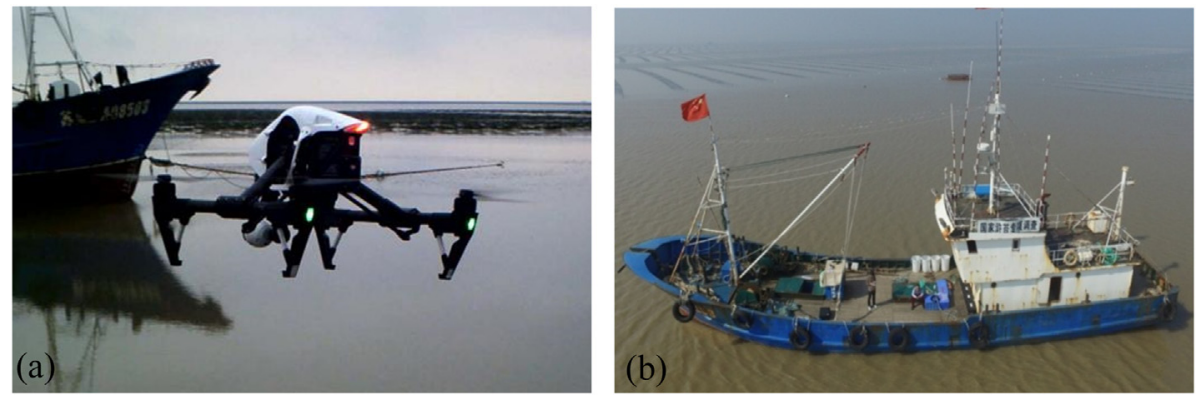

Fig. 2. (a) The unmanned aerial vehicle (UAV) and (b) the research vessel used in this study.

Table 1

Statistics of field survey in this research.

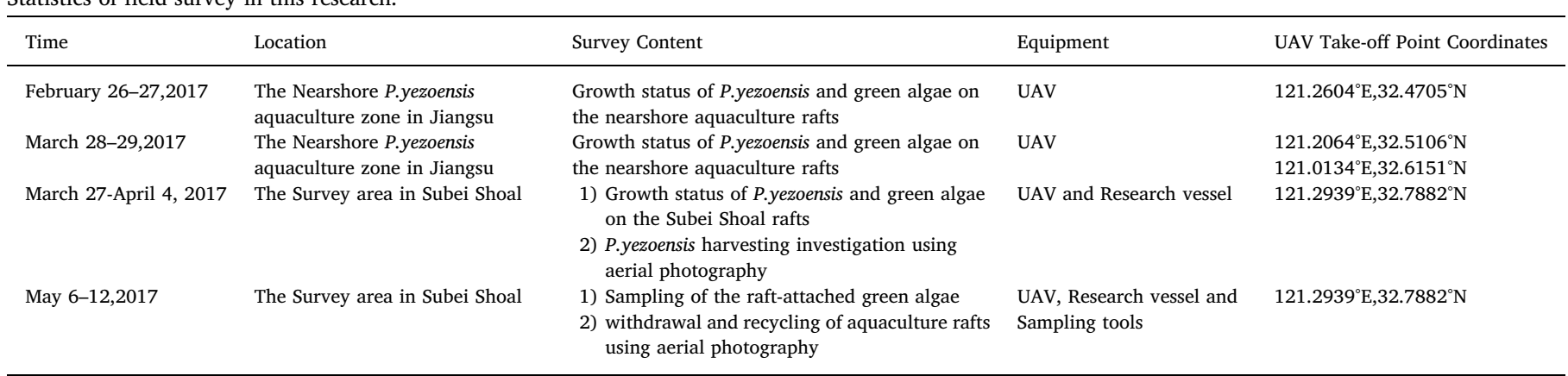

UAV: unmanned aerial vehicle.

artificial farming operations. Information relating to the field survey is shown in Table 1.

To extract the total aquaculture raft area of the Subei Shoal in 2017, multi-source satellite images including Gaofen-1 (GF-1) and Sentinel2A (S2A) were collected and adopted. GF-1 images were from the China Centre for Resources Satellite Data and Application, and had a $16 \mathrm{~m}$ spatial resolution and four day revisit cycle. S2A images (10 m spatial resolution) were provided by the European Space Agency. The downloaded satellite images were checked to identify days that were sufficiently cloud-free and to observe the rafts at high tide during the $P$. yezoensis growth period. For these reasons, the GF-1 images of the Subei Shoal on January 24 and March 2, 2017, and the S2A images on March 2, 2017, were selected. Multiple images from different satellites were used as a reference to ensure that the rafts were not missed. These satellite images were first processed by atmospheric correction and projection conversion. The $P$. yezoensis raft was then extracted by visual interpretation and manual vectorization.

We obtained statistical data from the China Fisheries Statistics Yearbook 2018 (Ministry of Agriculture and Rural Affairs of China, 2018), which is a compilation of statistical data relating to capture fisheries and aquaculture production, employment, commodities production, and trade etc. Specifically, we used yearbook data on total production and the total aquaculture area of $P$. yezoensis in Jiangsu province during 2017.

\subsection{Methods of biomass estimation}

The biomass estimation method for GAAN was based on the combination of data from the UAV, high-resolution satellite images, and the statistical yearbook. First, ratios of the area coverage of $P$. yezoensis to GAAN were calculated based on the UAV data. Then the distribution area of all rafts in the Subei Shoal was extracted from satellite data, and then the $P$. yezoensis production in the Subei Shoal in 2017 was estimated. The biomass of GAAN of the Subei Shoal in 2017 was then calculated (Fig. 3).

The biomass estimation method for GAAR was based on the high- resolution satellite data and field sampling data (Fig. 3). The size and areas of rafts, the number of nursery-nets, and the total length of each raft's rope, were measured from the satellite images. The total biomass of GAAR was calculated using data from these two sources.

\section{Results}

\subsection{Biomass estimation of GAAN}

\subsubsection{UAV images of algae in nursery-nets}

The digital orthophoto images of the rafts (Fig. 4a) generated by the UAV aerial survey data had a correct coordinate system, but the images after the mosaic appeared with a chromatic aberration, and the object features of some splicing areas were deformed (Fig. 4b). Thus, this affected the identification and extraction of area coverage of green algae and $P$. yezoensis. Therefore, single, clear, original JPG images along with the digital orthophoto images were used for image registration and overlay. The overlay images (an example is shown in Fig. 4c) were not only non-destructive but also within the correct coordinate system. The nursery-nets in the overlay images were subsequently clipped and the areas covered by $P$. yezoensis and GAAN in each of the 61 nursery-nets were extracted by means of the maximum likelihood classification. We used the green color to represent green algae, the magenta color to represent $P$. yezoensis (Fig. 4e). In addition, the original images (e.g., Fig. 4f) and the resultant images (e.g., Fig. 4g) were magnified to the same scale, and were then visually compared to check that the classification results were accurate.

There were 113568 pixels of GAAN and 49009 pixels of $P$. yezoensis in the classification results for the example provided in Fig. 4. The number of pixels was then multiplied by the image resolution to yield the area coverage of each type of algae. In the nursery-net example shown in Fig. 4e, the area of GAAN coverage was $0.17 \mathrm{~m}^{2}$ and the area of $P$. yezoensis coverage was $0.39 \mathrm{~m}^{2}$. The same procedure was applied to the remaining 60 nursery-nets, all 61 classified images of the nursery-nets can be found in Supplementary Table 1. The corresponding results are shown in Table 2. 


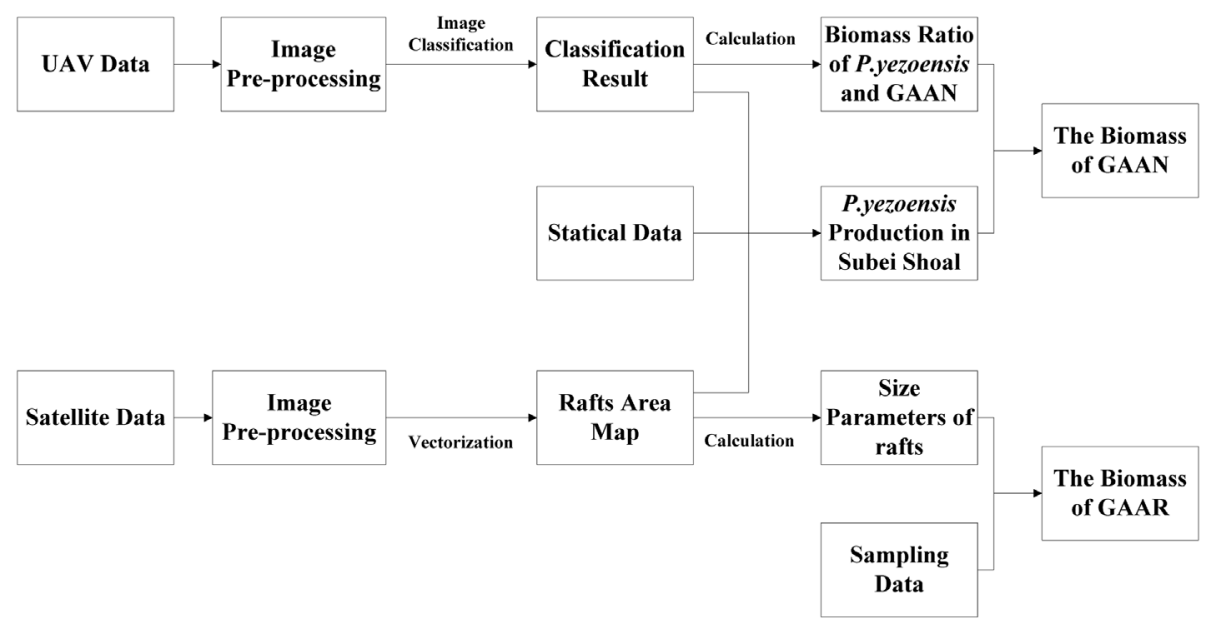

Fig. 3. Flow chart illustrating the two estimation methods for green algae attached to nursery-net (GAAN) and green algae attached to rope (GAAR).

For the 61 nursery-nets, the average areas of coverage for $P$. yezoensis and GAAN were $0.52 \mathrm{~m}^{2}$ and $0.85 \mathrm{~m}^{2}$, respectively. The ratio of the two was 0.61 , which was regarded as the biomass ratio $\left(\boldsymbol{R}_{1}\right)$ of $P$. yezoensis and GAAN on the aquaculture rafts in the Subei Shoal during 2017.

\subsubsection{Estimation of $P$. yezoensis production in the Subei Shoal}

According to the China Fisheries Statistics Yearbook 2018, the total production $\left(\boldsymbol{W}_{j s}\right)$ of $P$. yezoensis in Jiangsu province during 2017 was $41860 \mathrm{t}$, and the total aquaculture area $\left(S_{j s}\right)$ of $P$. yezoensis was 47255 ha. The $P$. yezoensis aquaculture area $\left(S_{s s}\right)$ in the Subei Shoal, which was extracted from satellite images, was 22907 ha. The accuracy of our calculation of $S_{s s}$ is supported by previous studies that reported that the aquaculture area of $P$. yezoensis in the Subei Shoal was 23333 ha (Yan et al., 2018). Other $P$. yezoensis aquaculture zones in Jiangsu province are closer to the shore that of those in the Subei Shoal, and have been collectively termed here as the nearshore $P$. yezoensis aquaculture zone. The corresponding area of the nearshore $P$. yezoensis, $\boldsymbol{S}_{\boldsymbol{n} \boldsymbol{s}}$, can be expressed by Eq. (1):

$S_{n s}=S_{j s}-S_{s s}$

In order to estimate the production of $P$. yezoensis in the Subei
Shoal, based on the known value of the total production of $P$. yezoensis in Jiangsu province and the ratio of the areas of the Subei Shoal and the nearshore $P$. yezoensis aquaculture zone, it is also necessary to consider the ratio of the per unit area yields (unit yield) of these two regions. Liu et al. (2017) found that in order to reduce the influence of green algae to $P$. yezoensis algae, the methods of artificial stripping or acid-coating were often adopted to remove GAAN in the Jiangsu nearshore aquaculture zones. However, in the Subei Shoal aquaculture zone, GAAN were not generally treated due to the distance of the aquaculture zone from the shore. Hence, it was understood that the biomass of GAAN in the Subei Shoal aquaculture zone was significantly higher than of that in the nearshore aquaculture zones (Liu et al., 2013a), which was verified by our field investigation. $P$. yezoensis algae in the Jiangsu nearshore aquaculture zones grow well and take advantage of nurserynets (Fig. 5), but in the Subei Shoal survey area, varying amounts of green algae were attached to most of the nursery-nets (Table 2).

Taking the data for the 61 nursery-nets sampled in our study (Table 2) as a sample set, we postulated that the biomass composition of the patch with the maximum coverage area of $P$. yezoensis (No. 35 in Table 2) would be quite similar to that of nursery-nets in the Jiangsu nearshore aquaculture zone. The ratio $\left(\boldsymbol{R}_{s}\right)$ of the $P$. yezoensis unit yield in the Subei Shoal aquaculture zone was calculated using the ratio of

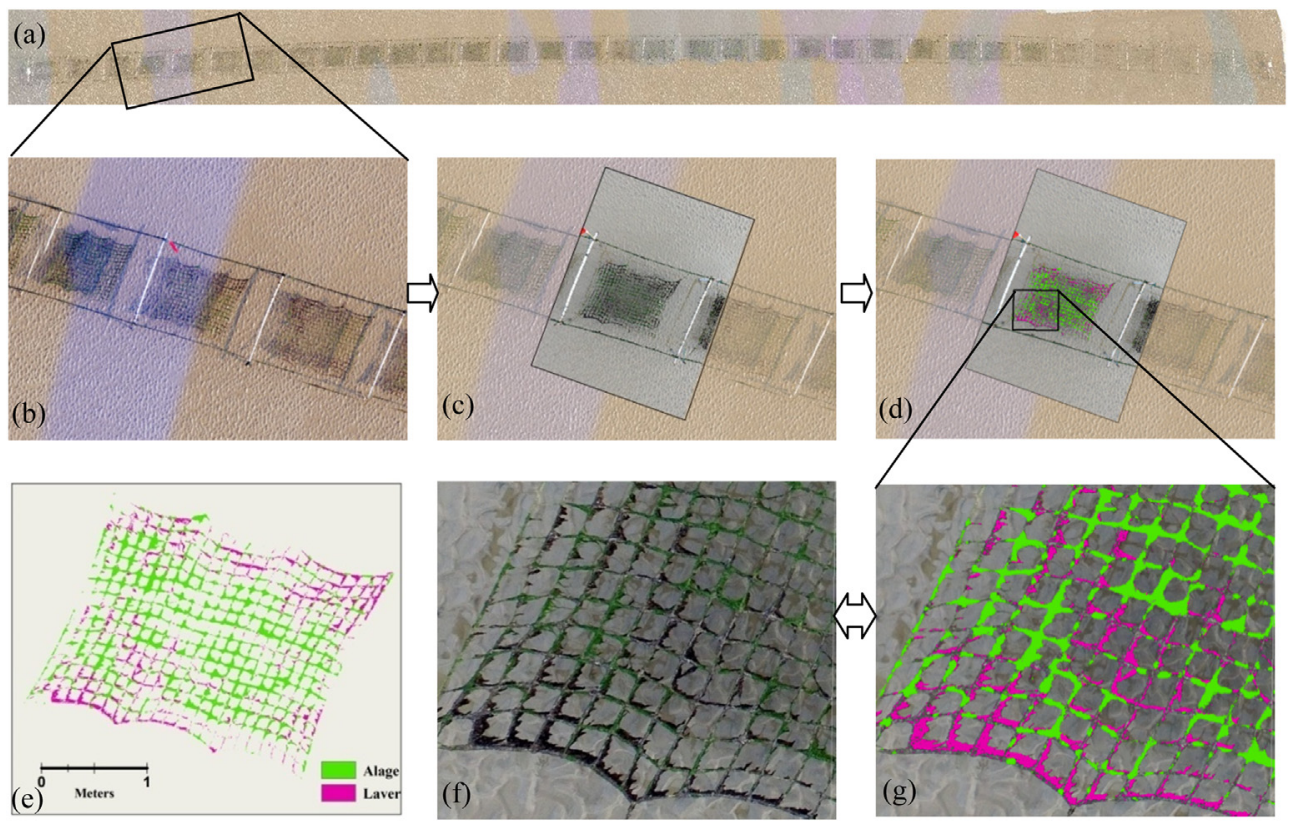

Fig. 4. (a) The orthophoto of rafts. (b-e) Classification procedure of green algae and $P$. yezoensis attached to nursery-nets. (f, g) Comparison of original and resultant images at the same scale for one example nurserynet. (For interpretation of the references to color in this figure legend, the reader is referred to the Web version of this article.) 
Table 2

The area $\left(\mathrm{m}^{2}\right)$ of $P$. yezoensis and GAAN on the 61 nursery-nets (where $\boldsymbol{P}$ is the area of $P$. yezoensis, and $\boldsymbol{G}$ is the area of GAAN).

\begin{tabular}{|c|c|c|c|c|c|c|c|c|c|c|c|c|c|c|c|c|}
\hline No. & 1 & 2 & 3 & 4 & 5 & 6 & 7 & 8 & 9 & 10 & 11 & 12 & 13 & 14 & 15 & 16 \\
\hline$P$ & 0.07 & 0.07 & 0.24 & 0.50 & 0.17 & 1.59 & 0.30 & 0.30 & 0.14 & 0.84 & 0.84 & 1.27 & 0.78 & 1.54 & 0.59 & 0.32 \\
\hline$G$ & 0.27 & 0.14 & 0.05 & 1.04 & 0.39 & 0.06 & 0.15 & 0.03 & 0.44 & 1.58 & 1.57 & 0.89 & 0.39 & 0.60 & 0.65 & 0.97 \\
\hline No. & 17 & 18 & 19 & 20 & 21 & 22 & 23 & 24 & 25 & 26 & 27 & 28 & 29 & 30 & 31 & 32 \\
\hline$P$ & 1.31 & 1.01 & 0.46 & 0.55 & 0.41 & 0.60 & 0.50 & 0.81 & 0.76 & 0.19 & 0.24 & 0.08 & 0.23 & 0.13 & 0.36 & 0.49 \\
\hline$G$ & 0.01 & 0.83 & 0.77 & 0.34 & 0.83 & 1.61 & 1.06 & 0.42 & 0.14 & 1.22 & 1.15 & 0.98 & 0.03 & 0.14 & 1.45 & 0.20 \\
\hline No. & 33 & 34 & 35 & 36 & 37 & 38 & 39 & 40 & 41 & 42 & 43 & 44 & 45 & 46 & 47 & 48 \\
\hline$P$ & 0.06 & 1.19 & 3.30 & 0.46 & 0.39 & 0.13 & 0.74 & 0.13 & 0.30 & 0.21 & 0.22 & 0.54 & 0.42 & 0.20 & 0.19 & 0.16 \\
\hline$G$ & 0.88 & 0.18 & 0.05 & 1.19 & 0.20 & 0.08 & 0.98 & 1.56 & 1.70 & 1.10 & 2.97 & 0.43 & 1.97 & 2.80 & 2.98 & 2.00 \\
\hline No. & 49 & 50 & 51 & 52 & 53 & 54 & 55 & 56 & 57 & 58 & 59 & 60 & 61 & & & \\
\hline$P$ & 0.14 & 0.64 & 0.40 & 0.54 & 0.12 & 0.21 & 0.19 & 0.05 & 0.41 & 0.02 & 0.60 & 1.22 & 0.24 & & & \\
\hline$G$ & 2.29 & 1.50 & 0.19 & 0.64 & 0.48 & 0.51 & 1.39 & 0.93 & 1.25 & 0.33 & 0.03 & 0.65 & 0.98 & & & \\
\hline
\end{tabular}
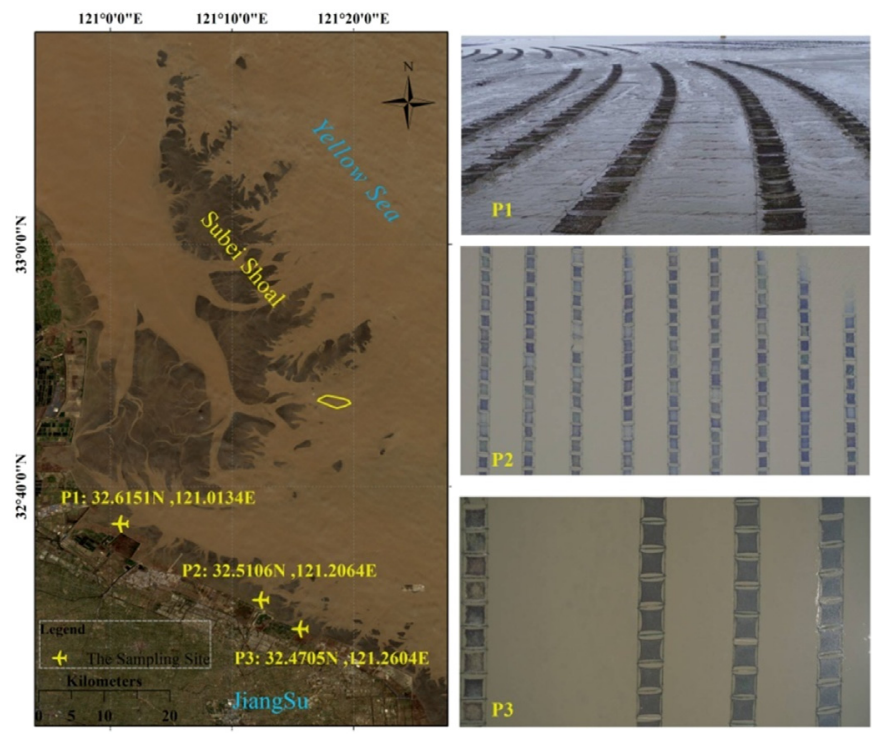

Fig. 5. Three survey sites (P1, P2, and P3) in the Jiangsu nearshore aquaculture zone where $P$. yezoensis grew well during March 29, 2017 (P1 and P2), and February 27, 2017 (P3).

the maximum $P$. yezoensis patch area (3.302, No. 35 in Table 2) of the 61 samples to the average area of the 61 samples (0.521), i.e., $\boldsymbol{R}_{\boldsymbol{s}} 6.34$.

The ratio $\left(\boldsymbol{R}_{\boldsymbol{t}}\right)$ of the total production of $P$. yezoensis algae in the Jiangsu nearshore zone to that in the Subei Shoal zone can be expressed by Eq. (2):

$R_{t}=\frac{S_{n s} * R_{s}}{S_{S s}}$

where $S_{n s}$ represents the area of the Jiangsu nearshore aquaculture zone, $S_{s s}$ represents the area of the Subei Shoal aquaculture zone, and $\boldsymbol{R}_{\boldsymbol{s}}$ represents the ratio of the $P$. yezoensis unit yield in the two zones.

The $P$. yezoensis total production $\left(\boldsymbol{W}_{s s}\right)$ in the Subei Shoal zone during 2017 can be expressed as Eq. (3):

$W_{s s}=\frac{W_{j s}}{R_{t}+1}$

where $\boldsymbol{W}_{j s}$ represents the $P$. yezoensis total production of Jiangsu province in 2017 from the statistical yearbook.

\subsubsection{Biomass estimation of GAAN in the Subei Shoal}

The biomass $\left(\boldsymbol{W}_{1}\right)$ of GAAN in the Subei Shoal can be expressed as Eq. (4):

$W_{1}=\frac{W_{S S}}{R_{1}}$

where $\boldsymbol{R}_{\boldsymbol{1}}$ represents the biomass ratio of $P$. yezoensis to green algae attached to aquaculture rafts in Subei Shoal (Section 3.1.1).

Based on the statistical yearbook data, and in consideration of the difference between the unit yield of $P$. yezoensis in the Jiangsu nearshore aquaculture zones and the Subei Shoal aquaculture zone, the total production $\left(\boldsymbol{W}_{s s}\right)$ of $P$. yezoensis in the Subei Shoal was calculate using Eq. (3) to be 5409 t. According to Eq. (4), the biomass $\left(\boldsymbol{W}_{\boldsymbol{1}}\right)$ of GAAN in the Subei Shoal during 2017 was calculated to be $8868 \mathrm{t}$.

\subsection{Biomass estimation of GAAR}

The distribution map of the rafts in the Subei Shoal is shown in Fig. 6 a. The total area covered by rafts $\left(\boldsymbol{S}_{s s}\right)$ in the Subei Shoal in 2017 was calculated to be 22907 ha. The smallest unit that can be distinguished from the satellite images was a row of rafts (e.g., yellow outlined area in Fig. 6b). The satellite images contained multi-column rafts and it was possible to observe the spacing among the columns as well as the spacing between the nursery-nets and the borders of raft units. The area between a nursery-net and the next nursery-net was removed in order to calculate the total number of nursery-nets in the Subei Shoal. The aquaculture rafts were distributed regularly and were all of a consistent size. The distribution pattern of a single row of raft is shown in Fig. 6d.

In a row of rafts, the proportion ( $\boldsymbol{r} 1)$ of the area occupied by the rafts is defined as:

$r 1=\frac{w * i}{w * i+a *(i-1)}$

where $\boldsymbol{i}$ represents the number of columns in a row of rafts.

When the value of $\boldsymbol{i}$ is large enough, $\boldsymbol{r} \mathbf{1}$ can take an approximation, as defined by Eq. (5):

$r 1 \approx \frac{w * i}{w * i+a * i}=\frac{w}{w+a}$

In a raft unit, the proportion ( $r 2)$ of the area occupied by the nursery-net is given by Eq. (6):

$r 2=\frac{x * y}{w * l}$

The total number $(\boldsymbol{N})$ of nursery-nets in the Subei Shoal can be expressed as Eq. (7):

$N=\frac{S_{s S} * r 1 * r 2}{x * y}$

The rope lengths $\left(\boldsymbol{L}_{\boldsymbol{g}}\right)$ on both sides of a raft unit can be expressed by Eq. (8):

$L_{g}=2 * l$

The total biomass $\left(\boldsymbol{W}_{2}\right)$ of GAAR in the $P$. yezoensis aquaculture raft in the Subei Shoal can be expressed by Eq. (9): 
(a) ${ }_{121^{\circ} 0^{\prime} 0 " \mathrm{E}}$ $121^{\circ} 10^{\prime} 0^{\prime \prime} \mathrm{E}$ $121^{\circ} 20^{\prime} 0 " \mathrm{E}$
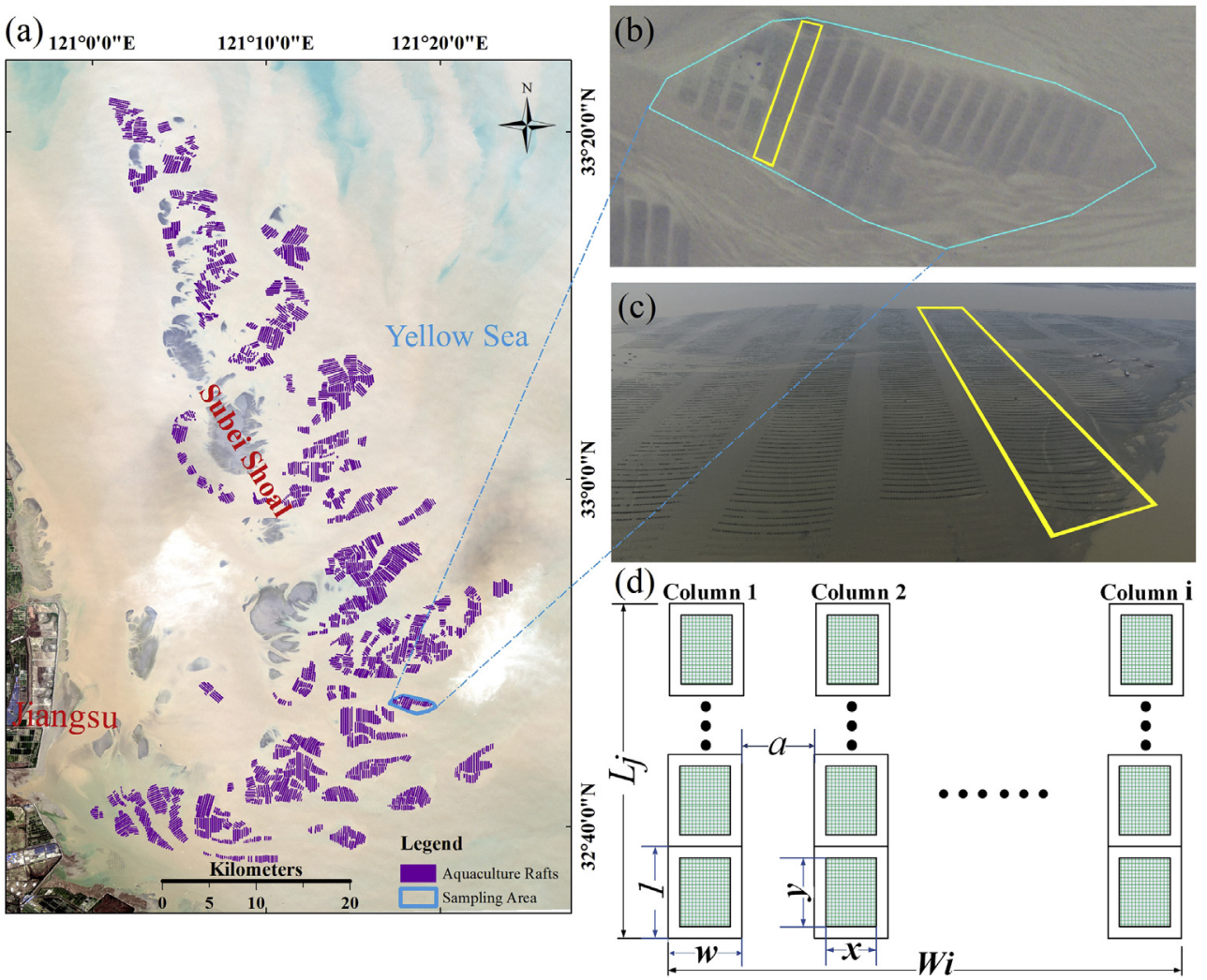

Fig. 6. (a) Distribution map of $P$. yezoensis aquaculture rafts in the Subei Shoal. (b) Satellite image of the sampling area. (c) Unmanned aerial vehicle image of the sampling area, where the area marked by the yellow box is the same row as that in (b). (d) Distribution pattern for a single row of $P$. yezoensis rafts, where $\boldsymbol{w}$ and $\boldsymbol{l}$ represent the width and length of a raft unit, respectively, $\boldsymbol{x}$ and $\boldsymbol{y}$ are the width and length of a nursery-net, respectively, and $\boldsymbol{a}$ represents the column spacing. From our field measurements: $\boldsymbol{w}=3 \mathrm{~m}, \quad \boldsymbol{l}=3.8 \mathrm{~m}, \quad x=2.2 \mathrm{~m}$, $\boldsymbol{y}=3 \mathrm{~m}$, and $\boldsymbol{a}=10 \mathrm{~m}$. (For interpretation of the references to color in this figure legend, the reader is referred to the Web version of this article.)
$W_{2}=\sum_{i=1}^{N}\left(L_{g} * W_{g}\right)$

where $\boldsymbol{W}_{\boldsymbol{g}}(\mathrm{g} / \mathrm{m})$ represents the mean wet weight of the GAAR (Section 2.1).

The total number $(\boldsymbol{N})$ of nursery-nets in the Subei Shoal was estimated from Eq. (7) to be 4636756 . The average wet weight $\left(\boldsymbol{W}_{\boldsymbol{g}}\right)$ of GAAR was $84.39 \mathrm{~g} / \mathrm{m}$ based on the 18 samples randomly chosen from the survey area. The biomass $\left(\boldsymbol{W}_{2}\right)$ of GAAR in the Subei Shoal was estimate using Eq. (9) to be $2974 \mathrm{t}$.

\section{Discussion}

\subsection{Uncertainties in biomass estimations}

Ideally, biomass estimations require validation and need to be assessed for uncertainties. In the case of the estimations of GAAN and GAAR, direct validation was not possible in this study. The accuracy of our biomass estimations could, however, have been affected by several factors. Firstly, when the UAV photographed the nursery-nets, it took the image from a vertical, downward viewpoint directly above the nursery-net. Since $P$. yezoensis and green algae on the nursery-nets also grow vertically downward, some of the algae would have been blocked in the image. In order to reduce possible errors associated with this, we adopted the following method. In addition to the official statistical data and the P.yezoensis aquiculture area in Subei Shoal extracted from the satellite images, the area of $P$. yezoensis and green algae extracted from the UAV orthophoto images were calculated as the ratio of the two, rather than using the actual values of the two directly.

Secondly, uncertainties exist since the survey area was only undertaken in the Zhugensha sandbank area of the Subei Shoal. Besides Zhugensha, the shoal also contains other sandbanks, such as Jiangjiasha, Gaoni and Dongsha (see Fig. 1b). Thus, the selected survey area was not necessarily representative of the entire shoal area. In addition, the growth and harvest period for $P$. yezoensis algae is from
December to April, whereas the aerial survey time of the nursery-nets was on March 30, 2017 (i.e., the end of the growth and harvest period). At this time of year, the unit yield of $P$. yezoensis algae may have been relatively low, which would result in the estimated total production $\left(\boldsymbol{W}_{s s}\right)$ to be lower than the actual value, and the biomass of GAAN $\left(\boldsymbol{W}_{\mathbf{1}}\right)$ to be higher than the actual value. Therefore, in future studies, the sampling area should cover more sandbanks. Sampling should also be undertaken every month during the growth period of $P$. yezoensis.

\subsection{New, high-accuracy biomass estimation method}

Satellite remote sensing technology has played an important role in assessing the origin, drift trajectories, and area distribution of the Yellow sea green-tides, and offers the advantages of being large scale, low cost, and enabling periodic observations (Hu et al., 2010; Cui et al., 2012). However, green algae do not fall into the sea to form large patches at the stage of being attached to rafts (see Section 4.3). The area of a single nursery-net of $P$. yezoensis is only $\sim 8-10 \mathrm{~m}^{2}$, which is much lower than the spatial resolution of available satellite data. As a result, it is impossible to observe green algae attached to rafts from satellite image. The UAV used in this study was small in size and maneuverable, and was able to generate orthophoto maps at a spatial resolution of a centimeter scale, which could identify $P$. yezoensis and green algae on the rafts. In comparison to satellite remote sensing, it broke the limits of cloud coverage and the revisit period, but its coverage was limited. Therefore, our study found that the combination of satellite and UAV data was successfully able to simultaneously meet the requirements of large coverage and high image resolution.

In this study, UAV was used to acquire highly accurate images at sea, and under more complex weather conditions than on land. The use of local fishing boats as the take-off and landing site allowed for the successful use of the UAV in our survey. The UAV thus provided the necessary aerial photography and large-scale mapping by aerial photogrammetry for the biomass estimations of GAAN and GAAR. The combined methods of satellite remote sensing, UAV, field survey, and 

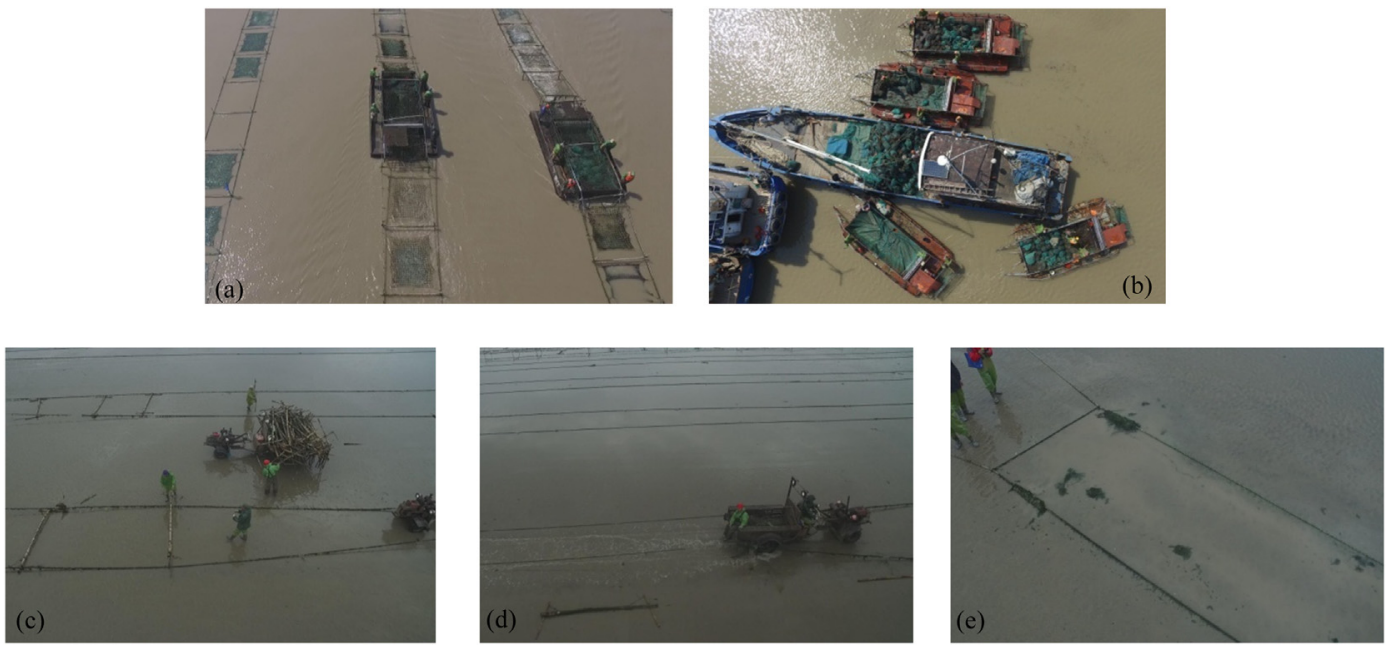

Fig. 7. Illustration showing the different processing methods for GAAN and GAAR: (a) GAAN being harvested with P. yezoensis; (b) GAAN and P. yezoensis being packed together; (c) withdrawal and recycling of aquaculture rafts, where green algae attached to bamboo poles is brought ashore; (d) GAAR being manually scraped off; (e) GAAR being abandoned on the tide flats. (For interpretation of the references to color in this figure legend, the reader is referred to the Web version of this article.)

statistical data proposed in this study provide a new, high-accuracy method for estimating attached macroalgal biomass at given locations.

\subsection{Implications for forecasting and prewarning}

As mentioned, GAAN can gather with P. yezoensis (Fig. 7a) during growth, and the two become packed together after harvesting and are shipped to the shore (Fig. 7b). GAAN is often artificially eliminated from the $P$. yezoensis at the processing plant, which therefore results in higher overall processing costs. Moreover, the seedling growth of GAAN have been found to reduce the yield and grade rate of $P$. yezoensis. Consequently, GAAN has reduced the economic benefits of the $P$. yezoensis aquaculture industry. After years of accumulating data on GAAN biomass, temperatures, seawater nutrients, and other variables related to the yield of $P$. yezoensis, the findings of this study therefore provide important reference data for the prediction of yield and income related to $P$. yezoensis aquaculture.

Under suitable environmental conditions, the micro-propagules of green algae can attach and germinate when they meet rafts used for growing $P$. yezoensis, thereby forming the green algae attached to rafts. During the raft withdrawal operation, GAAR is scraped off by a walking tractor (Fig. 7d) and then abandoned on the tidal flats (Fig. 7e). It can then float into the sea with the tide, and eventually forms the green-tide in the Yellow Sea (Liu et al., 2009). Current research found that the density of micro-propagules of green algae were closely related to the biomass of GAAR and the scale of green-tides. However, their direct contribution to green-tides in the Yellow Sea still requires support from in-situ data (Wang et al., 2018). The density of micro-propagules can be determined by laboratory culture (Liu et al., 2016), and the distribution area and biomass of a green-tide can be measured by satellite remote sensing and in-situ sampling. The biomass estimation method of GAAR proposed in the present study could provide the necessary data support for exploring the quantitative relationship between density of micropropagules, the distribution area of a green-tide, and the biomass of a green-tide. The proposed methods could also contribute to forecasts of the scale of green-tides, and assist in prewarning of green-tide disasters.

\section{Declaration of competing interest}

The authors declare that they have no known competing financial interests or personal relationships that could have appeared to influence the work reported in this paper.

\section{Acknowledgements}

We appreciated the comments from Dr. Pat Hutchings and also for her hard work on finalizing the manuscript. We also thank Dr. Paul Shin and two anonymous reviewers for valuable comments and suggestions which greatly improved the manuscript. This work was supported by the NSFC fund project (41876107), the NSFC-Shandong joint fund project (U1706219), the Basic Special Program of Ministry of Science and Technology (2014FY210600), and the Aoshan Science and Technology Innovation Program of Qingdao National Laboratory for Marine Science and Technology (2016ASKJ02).

\section{Appendix A. Supplementary data}

Supplementary data to this article can be found online at https:// doi.org/10.1016/j.marpolbul.2019.110678.

\section{References}

Cui, T.W., Zhang, J., Sun, L., Jia, Y.J., Zhao, W., Wang, Z.L., Meng, J.M., 2012. Satellite monitoring of massive green macroalgae bloom (GMB): imaging ability comparison of multi-source data and drifting velocity estimation. Int. J. Remote Sens. 33, 5513-5527.

Hu, C., Li, D., Chen, C., Ge, J., Muller-Karger, F.E., Liu, J., Yu, F., He, M.-X., 2010. On the recurrent ulva prolifera blooms in the Yellow sea and east China sea. J. Geophys. Res.: Oceans 115, C05017. https://doi.org/10.1029/2009JC005561.

Huo, Y., Han, H., Hua, L., Wei, Z., Yu, K., Shi, H., Kim, J.K., Yarish, C., He, P., 2016. Tracing the origin of green macroalgal blooms based on the large scale spatio-temporal distribution of Ulva microscopic propagules and settled mature Ulva vegetative thalli in coastal regions of the Yellow Sea, China. Harmful Algae 59, 91-99.

Liu, C., Ren, X., Peimin, H., Xiangsheng, C., Jinhui, W., Jingwen, L., Lingyun, X., 2013a. The Investigation and Study on the Origin of Green Tide in the South Yellow Sea, 2013 Shanghai-Busan Marine Forum Shanghai China.

Liu, C.C., Xu, R., He, P.M., Zhang, Z.L., Qin, Y.T., Xiang, L.Y., Deng, B.P., Liu, S.H., Ji, X., 2017. Research on the relations betwwn green tide and porphyra cultivation in the south Yellow Sea. Mar. Sci. 41, 35-43.

Liu, D., Keesing, J.K., He, P., Wang, Z., Shi, Y., Wang, Y., 2013b. The world's largest macroalgal bloom in the Yellow Sea, China: formation and implications. Estuar. Coast Shelf Sci. 129, 2-10.

Liu, D., Keesing, J.K., Xing, Q., Shi, P., 2009. World's largest macroalgal bloom caused by expansion of seaweed aquaculture in China. Mar. Pollut. Bull. 58, 888-895.

Liu, X., Wang, Z., Fan, S., Xiao, J., Yan, L.I., Zhang, X., Ruixiang, L.I., Wang, X., 2016. The current situations of green macroalgae and micro-propagules in Pyropia aquaculture of the Subei Shoal in spring of 2013. Acta Oceanol. Sin. 35, 94-100.

Liu, X., Yan, L., Wang, Z., Zhang, Q., Cai, X., 2015. Cruise observation of Ulva prolifera bloom in the southern Yellow Sea, China. Estuar. Coast Shelf Sci. 163, 17-22.

Lu, Q.Q., Zhou, W., Zhu, J.Y., Yan, B.L., Ni, J.Z., Yang, L.E., 2018. The History,Statue quo and development trend of pyropia yezoensis industry of China. Marine. Econ. China. 1, 3-11.

Ministry of Agriculture and Rural Affairs of China, N.F.T.E.C., China Society of Fisheries, 
2018. In: M.o.A.a.R.A.o.t.P.s.R.o (Ed.), China Fisheries Statistics Yearbook 2018, in: China. China Agriculture Press, BeiJing.

Nelson, T.A., Karalon, H., Nelson, A.V., Heather, R., Ruth, H., Alstyne, K.L., Van, Lee, B., Simunds, D.J., Kerri, F., 2008. Ecological and physiological controls of species composition in green macroalgal blooms. Ecology 89, 1287-1298.

Shi, M.J., Wang, H.C., 1996. Method for preventing green algae in artificial breeding period of Porphyra yezoensis. J. Aquacul. 2, 10-11.

Victor, S., Adriana, Z., 2013. Green and golden seaweed tides on the rise. Nature 504, $84-88$.

Wang, Z., Jie, X., Fan, S., Yan, L., Liu, X., Liu, D., 2015. Who made the world's largest green tide in China?-an integrated study on the initiation and early development of the green tide in Yellow Sea: green tide in Yellow Sea of China. Limnol. Oceanogr. 60, 1105-1117.

Wang, Z.L., Fu, M.Z., Xiao, J., Zhang, X.L., Song, W., 2018. Progress on the study of the Yellow Sea green tides caused by Ulva prolifera. Acta Oceanol. Sin. 40, 1-13.

Xiao, Y.F., Zhang, J., Cui, T.W., Gong, J.L., Xia, S.Z., Liu, R.J., Qing, P., Mu, B., 2017. Spectral characteristics and estimation models of floating green tide biomass on sea surface. Acta Opt. Sin. 37, 346-354.

Xu, F., Gao, Z., Jiang, X., Shang, W., Ning, J., Song, D., Ai, J., 2018. A UAV and S2A databased estimation of the initial biomass of green algae in the South Yellow Sea. Mar.
Pollut. Bull. 128, 408-414.

Yan, T., Yu, R.C., Zhou, M.J., Sun, S., 2018. Mechanism of massive formation and prevention strategy against large-scale green tides in the south Yellow Sea. Oceanol. Limnol. Sinica 49, 950-958.

Ye, N.H., Zhang, X.W., Mao, Y.Z., Liang, C.W., Xu, D., Zou, J., Zhuang, Z.M., Wang, Q.Y., 2011. 'Green tides' are overwhelming the coastline of our blue planet: taking the world's largest example. Ecol. Res. 26, 477-485.

Yu, R.C., Sun, S., Yan, T., Zhou, M.J., 2018. Progresses and perspectives on green-tide studies in the yellow sea. Oceanol. Limnol. Sinica 49, 942-949.

Zhang, C.B., Yang, S.T., Zhao, C.S., Lou, H.Z., Zhang, Y.C., Bai, J., Wang, Z.W., Guan, Y.B., Zhang, Y., 2018a. Topographic data accuracy verification of small consumer UAV. J. Remote Sense. 22, 185-195.

Zhang, Q.C., Kong, F.Z., Yan, T., Yu, R.C., Hu, X.K., Miao, H., Zhou, M.J., 2018b. Green algae detached from aquaculture rafts into seawater resulted in green tide occurrence in the yellow sea. Oceanol. Limnol. Sinica 49, 1014-1020.

Zhang, Q.C., Liu, Q., Yu, R.C., Kang, Z.J., Guo, W., Ding, Y.M., Chen, J.H., Wang, Y.F., Li, Y., Yan, T., 2015. Application of a fluorescence in situ hybridization (FISH) method to study green tides in the Yellow Sea. Estuar. Coast Shelf Sci. 163, 112-119.

Zhou, M.-J., Liu, D.-Y., Anderson, D.M., Valiela, I., 2015. Introduction to the special issue on green tides in the Yellow sea. Estuar. Coast Shelf Sci. 163, 3-8. 